Revue d'histoire de l'Amérique française

Z19 REVUE D.HISTOIRE DE L'AMÉRIQUE FRANÇAISE

\title{
Ordonnance pour la Subsistance du Corps de garde Le d. ${ }^{\text {er }}$ 10. ${ }^{\text {bre }} 1685$
}

\section{Fernand Lefebvre}

Volume 8, numéro 2, septembre 1954

URI : https://id.erudit.org/iderudit/301655ar

DOI : https://doi.org/10.7202/301655ar

Aller au sommaire du numéro

Éditeur(s)

Institut d'histoire de l'Amérique française

ISSN

0035-2357 (imprimé)

1492-1383 (numérique)

Découvrir la revue

Citer ce document

Lefebvre, F. (1954). Ordonnance pour la Subsistance du Corps de garde Le d. ${ }^{\text {er }}$ 10. ${ }^{\text {bre }}$ 1685. Revue d'histoire de l'Amérique française, 8(2), 260-261.

https://doi.org/10.7202/301655ar d'utilisation que vous pouvez consulter en ligne.

https://apropos.erudit.org/fr/usagers/politique-dutilisation/ 
ordonnance pour La Subsistance du Corps de garde Le d.er 10. bre 1685.*

SUR Ce qui Nous a esté representé par Le substi’ en Ce Bailliage que Nonobstant La publi'on que nous aurions faite par affiches pour faire assçavoir a tous Les habitans de la banlieue de Cette Ville que Sil y en avoit quelques uns deux qui voulleussent en Reprandre de traisner et voiturer Le bois Coupé pour Lentretien du feu du Corps de garde de Ce Lieu dans La forest de $\mathrm{M}^{\mathrm{rs}}$. Les Seig. ${ }^{\mathrm{rs}}$ de Cette Isle aud Corps de garde rue Saint Joseph en payant en argent Ce qui seroit raisonnable pour Leur peine et charroy Nauroient qua nous venir trouver pour en Convenir avec nous en nostre Logis Cependant aucuns desd habitans ny Seroient tenus despuis plus de quinze Jours que Lad. ${ }^{e}$ publi'on a esté faitte a la porte de leglise Issu de grande Messe par [ déchiré] bailly pourquoy les soldats qui Montent La garde soufriroient beaucoup de froid accause de la Rigueur de la saison en faisant le service du Roy pour Le bien et Conserva'on de la Collonnye a Ces Causes Mons. ${ }^{r}$ Le gouverneur pour Sa Majesté en Cetted ${ }^{\mathbf{e}}$ Isle Nous auroit dit en Conformitté des ordres de Monseig. ${ }^{\mathrm{r}}$ Le Marquis de denonville Gouverneur et Lieutenant general po[ ur ] Le Roy en Cè pais par lesquels

* Archives judiciaires de Montréal. - Ce document est à rattacher à notre "Histoire du Guet à Montréal" parue dans la Revue, livraison de septembre 1952. Transcription et communication de FERNAND LEFEBVRE. 
Il nous enjoignoit de trouver quelques Moyen de faire voiturer La quantitté de bois quil seroit Jugé Nesse' pour Lentretien du feu du Corps de garde en Ce lieu ny ayant point de fonds destinés a Cet esfect au soullagement des pouples et N'en ayant pas trouvé de plus Commode ny de plus Convenab' apres en avoir Conferé avec $\mathrm{m}^{\mathrm{r}}$ Dollier $\mathrm{p}^{\text {tre }}$ Superieur du Semina[ $\mathrm{i}$ ]re de Cotte ISle pour quilz ne fussent point chargez ny fouller que d'Impozer et Cottizer Ceux qui nont pas Les Commoditté de Loger Les trouppes du Roy a quelques Sommes pour Ce destiner a faire Charroyer Led. ${ }^{t}$ bois de Chauffage pour Le Corps de garde qui Sur Le report du S.r Bizart Major de Cette Isle nen ont plus que pour Ce Jour quil nous en est venu advertir de La part de Mond $\mathrm{S}^{\mathrm{r}}$. Le gouverneur dordonner a tous Ceux qui ont Coustume de Charroyer pour Le Roy en payant de Cette Ville a la Chine d [ e ] voiturer Les trente Cinq Cordes qui ont esté destinée pour Lentretien de ce feu et les Contraindre Comme Il est dit en payant Ce qui Sera Juste [ déchiré ] suivant L'uzage nous en Consequence des ordres tant [que] descript que de Vive Voix sur Le reffus Tacitte desd voituriers AVONS ordonné et ordonnons a Robert Cavellier dit desloriers, Urbain Texier dit La Vigne, Jean Leduc Jean descarry dit Lehou, Jacques Beauvais, hugue picard, La ve' Raclos, Louis Gervais, Jean Gasteau, Mathurin Jousset dit Laloire, Jean Roy et françois prudhomme Tous habitans dans La banlieu de lad [déchiré] Ville de voiturer et Charroyer Les trente Cinq Cordes de bois de la forest desd

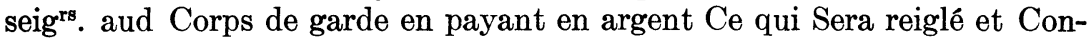
venu [ déchiré ] soit quilz [ en ] auront receu Les ordres par escript sur peine aux Contrevenans destre Contrains par touttes voys dires et raisons et Même d'amande Sy le Cas y eschoit mandons FAICT et Donné par Nous bailly de IIsle de montreal Le d'er Jour de decem ${ }^{\text {bre }}$ et de l'année mil six Cens quatre vingt Cinq

\section{Migeon de BRANSSAT}

\section{Bourgine}

La [ $\mathrm{n}$ ] mil Six cenS quatre Vaint Six et Le Neufieme Jour de Janvier avant midy Jay sergens Immatricullé au bailliage de Lille de montreal Soubsigné signifier Lordonnance Sy decus au nommés Jaque beauvexs An parlant a sca personne et an son domicille et a Jean Roy an parLant a Sca personne et a hugue pichart an parlant a Sa famme et a Jean gateau an parlant a Son fis et a La veuffe raclos an parLant a Sca personne et an Leur domicille et Leur ay faict commandement et par Vertu de La dicte ordonnance de charrier InceScamment Le bouest du corde et faute par eux di scatisfairre Leur ay declare quille seroit proteste contre euxs a telle Fain que de reSon a ce quille nen hignore

\section{G Quesnevillé}

leue peublie et affiche par moy Lory huissier Royal Immatriculle au bailliage de liSle de montreal le dimanche le trentiesme Jour de may a la porte de Lesglisse paroissialle de [illisible ] ville marie de montreal a LiSue de grande maiSSe [illisible ] 